\title{
Effect of Drugs on Chemoreceptor Responsiveness in Fetal Sheep
}

\author{
PETER FOCCO BOEKKOOI, JAN BAAN, JR., DAVID F. TEITEL, AND ABRAHAM M. RUDOLPH \\ Department of Pediatrics and Cardiovascular Research Institute, University of California at San \\ Francisco, San Francisco, California 94143
}

\begin{abstract}
This study was designed to examine the effects of the drugs ketamine, morphine, pentobarbital, and propranolol on fetal chemoreceptor responsiveness. Eleven fetal lambs (gestational age 125-133 d) were chronically instrumented with a catheter in a hindlimb artery and vein and a forelimb artery; a carotid arterial oximeter catheter was placed in six of these fetuses. An inflatable cuff occluder was placed around the maternal hypogastric artery. Acute fetal hypoxemia was induced repeatedly by reducing uterine blood flow. Fetal heart rate, arterial pressure, and carotid arterial oxygen saturation were monitored continuously before and after administering ketamine, morphine, pentobarbital, or propranolol to the fetus. The ratio $\Delta$ heart rate/ $\Delta \mathrm{O}_{2}$ saturation has been shown previously to be a reproducible index of chemoreflex
\end{abstract}

Acute fetal hypoxemia causes a bradycardic response (1), which we have previously shown is due to stimulation of peripheral chemoreceptors. The sensitivity of fetal chemoreceptors determines the severity of the bradycardic response. Chemoreceptor sensitivity depends on baseline oxygenation; the lower the baseline oxygenation, the more marked is the bradycardic response (2). Fetal chemoreceptor sensitivity may also change with the use of anesthetic agents; both baroreceptor function (3) and chemoreceptor function (4) in dogs are attenuated by pentobarbital. Fetal heart rate monitoring is frequently used for the assessment of fetal well being. Because drugs are often used during labor, it is important to know whether they influence chemoreceptor function, and thus alter the heart rate response to hypoxemia.

In this study, we examined the effect of ketamine, morphine, and pentobarbital and of a $\beta$-adrenoreceptor blocker (propranolol) on chemoreceptor responsiveness in unanesthetized fetal sheep. Chemoreceptor responsiveness was determined by measuring the heart rate response to acute hypoxemia. We have shown that the change of heart rate in response to a change in arterial oxygen saturation is an index of chemoreceptor re-

Received July 20, 1994; accepted July 10, 1995.

Correspondence and reprint requests: Abraham M. Rudolph, M.D., Box 0544, HSE 1403, University of California, San Francisco, San Francisco CA 94143-0544.

Supported in part by U.S. Public Health Service Grant HL 35842 and by the University of California, San Francisco Academic Senate Committee on Research. response. The differences in baseline values and changes with drugs were compared by multiple regression analysis coded by effects. Chemoreflex response was markedly attenuated by ketamine and morphine but not by pentobarbital or propranolol. Because the cardiovascular response to hypoxemia is blunted by some drugs, caution should be exercised in interpreting heart rate responses to hypoxemia in the fetus when these drugs have been administered to the mother. (Pediatr Res 38: 938-943, 1995)
Abbreviation
$\Delta \mathbf{H R} / \Delta \mathbf{s a t}=$ ratio of the decrease in heart rate to the decrease in $\mathrm{O}_{2}$ saturation

sponse to acute hypoxemia that is quantitative and reproducible (2).

\section{METHODS}

Surgical preparation. We studied 11 fetal sheep at gestational ages of 125-133 d. All procedures had been approved by the Committee on Animal Research at the University of California at San Francisco. Surgery was performed under aseptic conditions. Lumbar epidural anesthesia was achieved with 4 $\mathrm{mL}$ of $2 \%$ tetracaine (Pontocaine; Winthrop, New York). Ketamine $\mathrm{HCl}$ was inserted into a maternal venous catheter in doses of 100-150 $\mathrm{mg}$ every 10-15 min. We exposed the pregnant uterus through a midline incision in the maternal abdomen. We then exposed the fetal hindlimb through an incision in the uterus. Before each fetal skin incision, we injected lidocaine, $0.5 \%$, s.c. For measurement of pressures, we inserted polyvinyl catheters (inside diameter, $0.3 \mathrm{~mm}$; outside diameter, $0.5 \mathrm{~mm}$ ) via the femoral artery into the descending aorta and into the amniotic cavity (for zero pressure reference). For the administration of drugs, a catheter was inserted into the femoral vein and advanced to the inferior vena cava. After closure of the fetal skin and the uterus, the fetal head was exposed through another uterine incision. We inserted a catheter into the ascending aorta via the brachial artery, which we used to obtain blood samples for determination of $\mathrm{pH}, \mathrm{PCO}_{2}$, 
$\mathrm{Po}_{2}, \mathrm{Hb}$ concentration, and $\mathrm{Hb} \mathrm{O}_{2}$ saturation. In 6 of the 11 fetuses, we inserted another catheter into the ascending aorta via the carotid artery for the continuous measurement of $\mathrm{O}_{2}$ saturation (Abbott Critical Care, Mountain View, CA). After closure of the skin incision, warm $0.9 \% \mathrm{NaCl}$ solution was poured into the amniotic cavity to replace lost amniotic fluid. After closure of the uterine incision, we placed an inflatable balloon occluder around the maternal common hypogastric artery to regulate uterine blood flow. All catheters were exteriorized at the ewe's flank and placed in a pouch attached to the flank. During surgery and on each consecutive day, we administered antibiotics (100 mg of gentamicin and $2.10^{6} \mathrm{U}$ of penicillin) i.v. to the ewe and into the amniotic cavity. Experiments were performed 1-6 d after surgery.

Methods of measurement. The ewe was placed in a study cage and allowed free access to alfalfa pellets and water. Before the experiment, we connected catheters to Statham $\mathrm{P} 23 \mathrm{Db}$ strain-gauge transducers (Statham Instruments, Oxnard, CA), and (in six fetal lambs) the $\mathrm{O}_{2}$ saturation catheter to an $\mathrm{SO}_{2} / \mathrm{CO}$ Oximetrix computer (Abbott Critical Care, Mountain View, CA). The various signals were recorded continuously on a direct-writing polygraph (AstroMed, West Warwick, RI). Fetal heart rate was derived from the arterial pressure pulse, which triggered a cardiotachometer. Hb concentration and $\mathrm{Hb} \mathrm{O}_{2}$ saturation (Radiometer OSM2 hemoximeter, Copenhagen, Denmark) and $\mathrm{pH}, \mathrm{PCO}_{2}$, and $\mathrm{Po}_{2}$ were determined from ascending aortic blood samples (Corning $158 \mathrm{pH} / \mathrm{blood}$ gas analyzer, Medfield, MA). In the six fetuses equipped with the $\mathrm{O}_{2}$ saturation catheter, the catheter calibration was confirmed by the $\mathrm{O}_{2}$ saturation determined simultaneously in a blood sample.

For fetal administration of drugs, we used the following doses: ketamine, $3 \mathrm{mg} / \mathrm{kg}$ estimated fetal body weight; morphine, $0.1 \mathrm{mg} / \mathrm{kg}$; pentobarbital, $5 \mathrm{mg} / \mathrm{kg}$; and propranolol, 0.3 $\mathrm{mg} / \mathrm{kg}$. These doses were selected as those generally administered to newborn infants. We have also previously used these doses of the drugs in lambs to produce a pharmacologic effect.

Experimental protocol. We produced acute fetal hypoxemia $(<2 \mathrm{~min})$ by inflating the balloon occluder around the maternal common hypogastric artery. Within 5-30 s, fetal carotid arterial $\mathrm{O}_{2}$ saturation started to fall (Fig. 1). Heart rate began to fall $10-20 \mathrm{~s}$ after the decrease in $\mathrm{O}_{2}$ saturation, then reached a nadir (Fig. 1). After this nadir was reached, we released the balloon occluder. Before each occlusion, and at the heart rate nadir, we withdrew blood samples from the ascending aorta for the determination of blood gases. All analyses were done using $\mathrm{O}_{2}$ saturations measured in blood samples. After each occlusion, the fetuses were allowed to recover for about 10-15 min before further study. In 3 of the 11 fetuses, the balloon occluder did not function; therefore, we produced hypoxemia using an alternative method. A transparent plastic bag was placed around the ewe's head, and the bag inflated with a mixture of $4 \% \mathrm{CO}_{2}$ in nitrogen over a 1 -min period. This produced a decrease in $\mathrm{O}_{2}$ saturation and changes in other blood gases comparable to the effects after uterine arterial occlusion. No significant differences in the responses could be detected between the two techniques for producing hypoxemia.

After baseline experiments were performed, the studies were repeated $10 \mathrm{~min}$ after injection of one of the four drugs (ketamine, morphine, pentobarbital, or propranolol). In some fetuses, more than one drug was used. After the use of pentobarbital, we waited until the next day before performing new experiments. After injecting one of the other three drugs, we waited at least $3 \mathrm{~h}$ before we performed further studies. Ketamine was administered to nine fetuses, morphine to five, pentobarbital to five, and propranolol to seven fetuses (Table 1 ). Ketamine was given a total of 32 times, morphine 22, pentobarbital 20, and propranolol 16 times.

Data analysis. Chemoreceptor responsiveness was calculated as the ratio of the decrease in heart rate to the decrease in $\mathrm{O}_{2}$ saturation $(\Delta \mathrm{HR} / \Delta$ sat) during acute hypoxemia. Because the number of uterine arterial occlusions in each fetus varied (3-21 in the control group), we applied multiple linear regression analyses using dummy variables coded by effect $(5,6)$ to remove the effects of inter-animal variability. Using effects coding, the intra-animal variability is considered entirely within the set of dummy variables coding the individual sheep, as presented in Equation 1 below. Thus, large differences in the

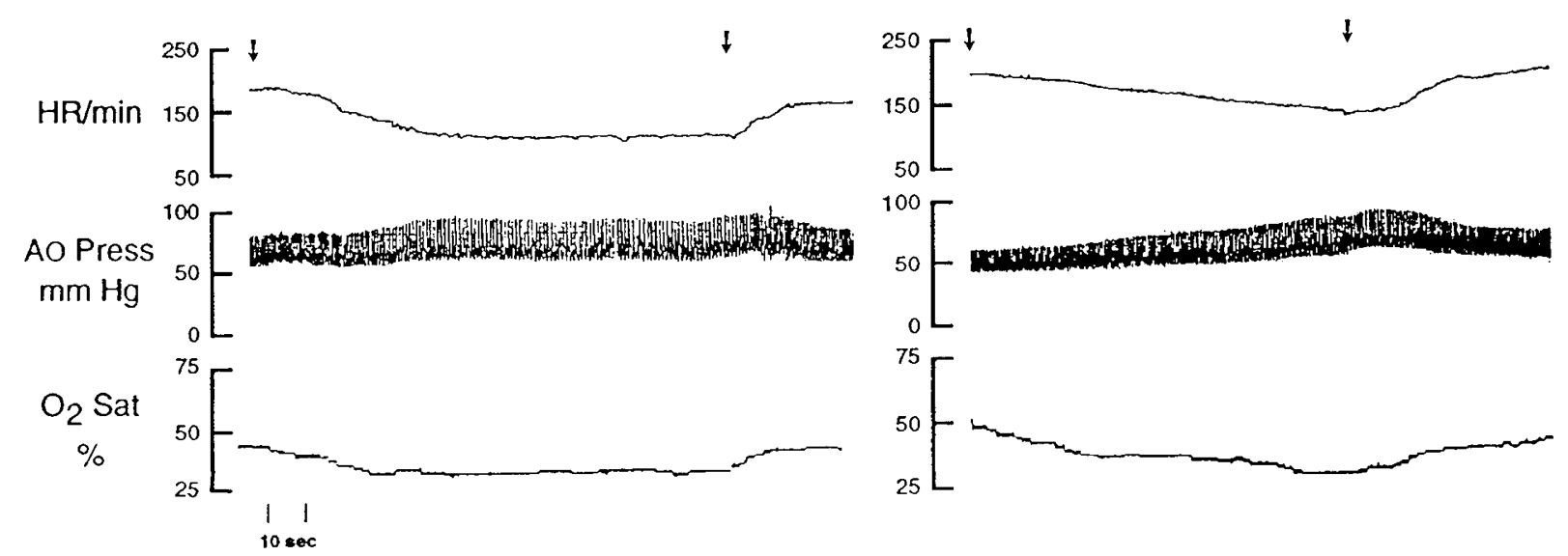

Figure 1. The responses of heart rate, aortic pressure, and carotid arterial oxygen saturation to acute hypoxemia are shown in a fetal lamb before (left) and after (right) administration of morphine to the fetus. Note that, in the control period, a relatively small fall in oxygen saturation is associated with a marked drop in heart rate. After morphine administration, a greater fall in oxygen saturation results in a slower and lesser decrease in heart rate. Arrows indicate initiation and termination of maternal hypogastric arterial compression. 
Table 1. Baseline variables and their changes during hypoxia, and the ratio $\Delta H R / \Delta$ sat mean $\pm S D$

\begin{tabular}{|c|c|c|c|c|c|}
\hline Variable & No drugs & Ketamine & Morphine & Pentobarbital & Propranolol \\
\hline Baseline $\mathrm{O}_{2}$ sat $(\%)$ & $63.5 \pm 11.7$ & $64.4 \pm 11.4$ & $63.5 \pm 6.9$ & $61.8 \pm 8.8$ & $66.9 \pm 12.0$ \\
\hline$\Delta \mathrm{O}_{2}$ sat $(\%)$ & $-26.3 \pm 8.0$ & $-30.2 \pm 8.7 *$ & $-32.8 \pm 9.2$ & $-29.5 \pm 10.8$ & $-27.9 \pm 6.4$ \\
\hline Baseline heart rate (beats/min) & $172 \pm 20$ & $188 \pm 38^{*}$ & $166 \pm 11 *$ & $201 \pm 29 *$ & $150 \pm 16^{*}$ \\
\hline$\Delta$ Heart rate (beats/min) & $-63 \pm 27$ & $-33 \pm 48^{*}$ & $-33 \pm 21 *$ & $-64 \pm 24$ & $-46 \pm 23$ \\
\hline Ratio $\Delta \mathrm{HR} / \Delta$ sat & $2.6 \pm 1.4$ & $1.2 \pm 1.8^{*}$ & $1.1 \pm 0.8^{*}$ & $2.2 \pm 0.6$ & $1.7 \pm 0.9$ \\
\hline Baseline $\mathrm{PCO}_{2}(\mathrm{kPa})$ & $6.71 \pm 0.85$ & $6.50 \pm 0.49$ & $6.73 \pm 0.72$ & $6.53 \pm 0.67$ & $6.80 \pm 0.84$ \\
\hline$\Delta \mathrm{PCO}_{2}(\mathrm{kPa})$ & $0.32 \pm 0.49$ & $0.48 \pm 0.48$ & $0.54 \pm 0.46$ & $0.52 \pm 0.51$ & $0.41 \pm 0.36$ \\
\hline Baseline pH & $7.37 \pm 0.05$ & $7.38 \pm 0.04$ & $7.36 \pm 0.05$ & $7.37 \pm 0.02$ & $7.38 \pm 0.04$ \\
\hline$\Delta \mathrm{pH}$ & $-0.01 \pm 0.02$ & $-0.01 \pm 0.02$ & $-0.01 \pm 0.03$ & $-0.01 \pm 0.02$ & $-0.01 \pm 0.01$ \\
\hline Baseline mean arterial pressure $(\mathrm{mm} \mathrm{Hg})$ & $57 \pm 9$ & $59 \pm 10$ & $52 \pm 3^{*}$ & $59 \pm 7^{*}$ & $57 \pm 6$ \\
\hline$\Delta$ mean pressure $(\mathrm{mm} \mathrm{Hg})$ & $1 \pm 6$ & $4 \pm 7 *$ & $4 \pm 5$ & $1 \pm 5$ & $0 \pm 5$ \\
\hline
\end{tabular}

* The coefficient of the effect of that drug is significant by multiple linear regression (see Table 2 for $p$ levels). The $n$ of the heart rate and saturation data are $189,35,21,20$, and 13 for no drugs, ketamine, morphine, and propranolol, respectively, whereas the $n$ of $\mathrm{pH}$ and $\mathrm{PCO}_{2}$ data are $150,34,18,15$, and 12 .

number of occlusions between animals for a given drug state do not impact on the predicted effect of that drug on the dependent variable of interest (such as $\Delta \mathrm{HR} / \Delta$ sat). Standard analysis of variance or multiple regression coded by reference are not possible in the presence of these large differences because the impact of one animal on the dependent variable would be greater than another.

From previous studies in our laboratory, we know that there is a significant inverse relationship between resting $\mathrm{O}_{2}$ saturation and the heart rate response to hypoxemia (2). Therefore, to test the effect of the various drugs on chemoreceptor responsiveness, we included oxygen saturation as an independent variable in the regression equation:

$$
Y=b_{0}+b_{s} S+\sum_{i=1}^{4} b_{g_{i}} G_{i}+\sum_{i=1}^{10} b_{f_{i}} F_{i}
$$

in which the dependent variable $Y$ is the ratio of $\Delta H R / \Delta$ sat, and $b_{0}$ the intercept of the equation representing the overall mean ratio when the predicted saturation is 0 . The continuous variable $S$ represents the effect of resting $\mathrm{O}_{2}$ saturation; $b_{s}$ is its coefficient of effect, estimating of the effect of a $1 \%$ change in resting saturation on the ratio. $G_{i}$ is the set of four dummy variables representing the four drug conditions (ketamine, morphine, pentobarbital, and propranolol) and control. This set of dummy variables is constructed so that each of the four drugs is associated with a separate coefficient, $b_{g_{i}}$. All ketamine occlusions were coded as $(1,0,0,0)$, pentobarbital occlusions as $(0,1,0,0)$, morphine occlusions as $(0,0,1,0)$, propranolol occlusions as $(0,0,0,1)$, and control occlusions as $(-1,-1,-1,-1)$. Thus, for example, doubling the value of the first $b_{g_{i}}$ ] predicts the change in $\Delta \mathrm{HR} / \Delta$ sat from control induced by the administration of ketamine. The set of variables $F_{i}$ represents the 10 dummy variables coding the 11 fetuses, also by effect (5).

We used $\mathrm{O}_{2}$ saturation rather than $\mathrm{Po}_{2}$ in the regression equation because, in the six fetuses with $\mathrm{O}_{2}$ saturation catheters, we were able to measure $\mathrm{O}_{2}$ saturation continuously and to follow the pattern of response accurately. In the range of saturations which occurred in this study, the $\mathrm{Hb}$ oxygen dissociation curve is quite linear, and thus initial analysis of $\Delta \mathrm{HR} / \Delta$ sat and $\Delta \mathrm{HR} / \Delta \mathrm{Po}_{2}$ yielded identical statistical results.
Regression equations were also constructed for the other dependent variables of interest (baseline and change in heart rate, $\mathrm{O}_{2}$ saturation, $\mathrm{PCO}_{2}, \mathrm{pH}$, and mean arterial pressure). These equations were similar to that of Equation 1 except that the continuous variable $S$ was not included, because it either covaried with the dependent variable (baseline and change in $\mathrm{O}_{2}$ saturation) or had no effect on the dependent variable (baseline and change in heart rate, $\mathrm{PCO}_{2}, \mathrm{pH}$, and mean arterial pressure).

All descriptive data are presented as mean \pm SD. Results of the regression equations are presented as the value of the coefficient \pm SEM.

\section{RESULTS}

Resting $\mathrm{O}_{2}$ saturation was normal during the control period $(63.5 \pm 11.7 \%)$ and did not vary among all conditions (Table 1). Baseline $\mathrm{PCO}_{2}$ and $\mathrm{pH}$ did not differ among all five conditions, and changes in each during the occlusions were small and statistically insignificant by regression analysis.

During control measurements, hypoxemia decreased $\mathrm{O}_{2}$ saturation by $26.3 \pm 8.0 \%$ and heart rate by $63 \pm 27$ beats/min, resulting in a mean $\Delta \mathrm{HR} / \Delta$ sat of $2.6 \pm 1.4$. Blood pressure did not change significantly.

Ketamine significantly increased baseline heart rate from $172 \pm 18$ to $189 \pm 35$ beats $/ \mathrm{min}$. During acute hypoxemia, heart rate decreased less despite a greater decrease in $\mathrm{O}_{2}$ saturation, resulting in a significantly lower $\Delta \mathrm{HR} / \Delta$ sat $(1.2 \pm$ 1.8 ). By regression analysis, $\Delta H R / \Delta$ sat is only $49 \%$ of control at a predicted saturation of $65 \%$ (Table 2). During the occlusion, blood pressure increased by $4 \pm 7 \mathrm{~mm} \mathrm{Hg}$.

Morphine administration was likewise associated with a greater decrease in $\mathrm{O}_{2}$ saturation and a lesser decrease in heart rate during acute hypoxemia (Table 1). This corresponded to a significantly lower $\Delta \mathrm{HR} / \Delta$ sat, predicted to be $54 \%$ of control at a saturation of $65 \%$ (Table 2). Mean arterial blood pressure did not change significantly during hypoxemia (Table 1).

Pentobarbital administration was associated with an increase in baseline heart rate to $201 \pm 29$ beats/min (Table 1). The decrease in $\mathrm{O}_{2}$ saturation and heart rate were not different from control, and thus $\Delta \mathrm{HR} / \Delta$ sat was not significantly different (Table 2). Blood pressure did not change significantly during hypoxemia. 
Table 2. Coefficients $(b, \pm S E)$ or multiple regression analysis of the ratio $\Delta H R / \Delta$ sat for the continuous variable $\mathrm{S}$ (baseline $\mathrm{O}_{2}$ saturation) and the dummy variables representing the effect of each drug, $G_{i}$; and the predicted ratio $\Delta H R / \Delta$ sat, for each drug and the control state at an $\mathrm{O}_{2}$ saturation of $65 \%$

\begin{tabular}{lccc}
\hline \multicolumn{1}{c}{ Variable } & $b$ (beats $/ \mathrm{min} / \mathrm{O}_{2}$ sat) & $p$ value & $\begin{array}{c}\Delta \mathrm{HR} / \Delta \text { sat } \\
(65 \%)\end{array}$ \\
\hline$S$ (baseline $\mathrm{O}_{2}$ saturation) & $-0.050 \pm 0.07$ & $<0.0001$ & \\
$G_{1}$ (ketamine) & $-0.607 \pm 0.171$ & $<0.0005$ & 1.294 \\
$G_{2}$ (morphine) & $-0.486 \pm 0.202$ & 0.017 & 1.415 \\
$G_{3}$ (pentobarbital) & $0.169 \pm 0.21$ & 0.424 & 1.99 \\
$G_{4}$ (propranolol) & $0.199 \pm 0.248$ & 0.424 & 2.1 \\
Control & & & 2.626 \\
\hline
\end{tabular}

The regression equation was statistically significant $(p<0.0001)$, and had an $r$ value of 0.76 . The $p$ value is of the corresponding $F$ test of each coefficient.

Propranolol administration was associated with a decrease in baseline heart rate to $150 \pm 16$ beats/min (Table 1 ). There were no other differences from control in the decrease in saturation or heart rate and thus $\Delta \mathrm{HR} / \Delta$ sat during hypoxemia, and blood pressure also did not change.

\section{DISCUSSION}

A bradycardic response to acute hypoxemia in the fetus has been well documented $(1,7,8)$. We have previously shown that the magnitude of this response was related to resting level of arterial $\mathrm{O}_{2}$ saturation increasing with decreasing control $\mathrm{O}_{2}$ saturation; it represents an index of chemoreceptor sensitivity (2). In the present study, we have shown that fetal administration of two different anesthetic agents, ketamine and morphine, depress this response, suggesting that each of these agents depresses chemoreceptor sensitivity.

To determine chemoreceptor sensitivity, we calculated $\Delta \mathrm{HR} / \Delta$ sat during acute hypoxemia induced by occlusion of the uterine artery (2). This response is assumed to reflect peripheral chemoreceptor function because it is abolished both by administration of atropine and by denervation of the aortic and carotid chemoreceptors (9). A significant concomitant effect of baroreceptor stimulation on heart rate was excluded in our study because changes in fetal arterial pressure during acute hypoxemia were trivial in all conditions, and significant only during ketamine administration. A similar mean blood pressure elevation $(5 \mathrm{~mm} \mathrm{Hg}$ ) by phenylephrine injection decreases heart rate by less than 5 beats/min (10), whereas heart rate decreased 6-fold more than that in response to acute hypoxemia after ketamine infusion. This index of carotid and aortic chemoreceptor sensitivity $(\Delta H R / \Delta$ sat) has considerable advantages over previous methods, such as recordings of carotid nerve activity, because it does not require anesthesia and exteriorization of the fetus, and because it can be used repeatedly in the same fetus in utero.

Anesthetics could depress peripheral chemoreceptor responsiveness by various mechanisms. Heart rate is regulated by both sympathetic and parasympathetic influences. In the hypoxic fetal lamb, blockade of parasympathetic regulation by atropine produces a greater effect on heart rate than does sympathetic blockade with propranolol (8). One mechanism by which anesthetic drugs may alter chemoreceptor response is by depressing the afferent component, either in the chemoreceptor cells, in the afferent nerve, or at the synaptic site. No evidence is available to support this hypothesis. A second mechanism is that the drug could affect basal sympathetic or parasympathetic tone. Ketamine has been reported to increase sympathetic activity by stimulating nonopioid phencyclidine/sigma receptors (11). If resting sympathetic activity is increased, the vagal stimulation resulting from hypoxemia will not cause as great a reduction in heart rate.

Morphine also has a stimulatory effect on the circulation when administered to conscious pigs (12) and cats (13). There is evidence that this effect is due to stimulation of hypothalamic nuclei, which leads to increased sympathetic activity (14). Thus, morphine could decrease the heart rate response to chemoreceptor stimulation in a manner similar to ketamine.

Although we did not measure peripheral vascular resistance, blood pressure increased to a greater extent during hypoxemia after ketamine administration than in the other conditions, although this increase was still quite small. This could be related to an increase in baseline sympathetic tone on the peripheral circulation.

The chemoreflex could be modified by a central effect of the drug. Pentobarbital is known to decrease both baroreflex (3, 15) and chemoreflex function in adult dogs (4). It is also known to increase baseline heart rate, which is in accordance with our findings in fetal sheep. However, plasma levels of catecholamines are not increased during pentobarbital administration; also, pentobarbital decreases the catecholamine response to baroreceptor stimulation (3). We cannot explain the lack of effect of pentobarbital on the hypoxemic response of the fetus as compared with the adult. It is possible that the postnatal effect of pentobarbital is centrally mediated and that this effect is abolished in the fetus because of the greater activity of pontine inhibitory areas in the brain on central chemoreceptor responses (16).

Administration of propranolol did not affect the heart rate response after chemoreceptor stimulation. Although it does reduce resting sympathetic tone, it did not significantly alter the response.

The observation that the bradycardic response to hypoxemia is greatly attenuated with morphine and ketamine administration has possible clinical implications. Fetal heart rate monitoring is an important tool in the assessment of fetal well being. During labor, opioids frequently have been used to relieve pain. These opioids cross the placenta and decrease fetal heart rate variability (8). Our data suggest that opioids as well as other drugs may also attenuate the fetal chemoreceptor response to hypoxemia. Because less bradycardic response occurs after administration of some drugs, it is suggested that their possible influence on signs of distress in the human fetus, such as heart rate decelerations, be explored. Similarly, because these drugs may be administered to newborn infants, it would be important to examine whether they may modify usual responses to hypoxia. 
Acknowledgment. We thank Christine Roman for excellent technical assistance.

\section{REFERENCES}

1. Itskovitz J, Goetzman BW, Rudolph AM 1982 The mechanism of late deceleration of the heart rate and its relationship to oxygenation in normoxemic and chronically hypoxemic fetal lambs. Am J Obstet Gynecol 142:66-73

2. Boekkooi PF, Baan Jr J, Teitel D, Rudolph AM 1992 Chemoreceptor responsiveness in fetal sheep. Am J Physiol 263:H162-H167

3. Zimpfer M, Manders WT, Barger AC, Vatner SF 1982 Pentobarbital alters compensatory neural and humoral mechanisms in response to hemorrhage. Am J Physiol 243:H713-H721

4. Zimpfer MS, Sit SP, Vatner SF 1981 Effects of anesthesia on the canine carotid chemoreceptor reflex. Circ Res 48:400-406

5. Glantz SA, Slinker BK 1990 Primer of Applied Regression and Analysis of Variance. McGraw-Hill, New York

6. Slinker BK, Glantz SA 1990 Missing data in two-way analysis of variance. Am J Physiol 258:R291-R297

7. Martin Jr CB, de Haan J, van der Wildt B, Jongsma HW, Dieleman A, Arts THM 1979 Mechanisms of late decelerations in the fetal heart rate. A study with autonomic blocking agents in fetal lambs. Eur J Obstet Gynecol Reprod Biol 9:361-373
8. Parer JT, Krueger TR, Harris JL 1980 Fetal oxygen consumption and mechanisms of heart rate response during artificially produced late decelerations of fetal heart rate in sheep. Am J Obstet Gynecol 136:478-482

9. Itskovitz J, Rudolph AM 1982 Denervation of arterial chemoreceptors and baroreceptors in fetal lambs in utero. Am J Physiol 242:H916-H920

10. Baan Jr J, Boekkooi PF, Teitel DF, Rudolph AM 1993 Heart rate fall during acute hypoxemia: a measure of chemoreceptor response in fetal sheep. $J$ Dev Physio 19:105-111

11. Seth S, Mukherjee D, Choudhary AK, Sinha JN, Gurtu S 1990 Opioid and non-opioid central cardiovascular effects of ketamine. Naunyn-Schmiedebergs Arch Pharmaco 342:535-538

12. Hannon JP, Bossone CA 1991 Cardiovascular and pulmonary effects of morphine in conscious pigs. Am J Physiol 261:R1286-R1293

13. Kayaalp SO, Kaymakcalan SA 1966 A comparative study of the effects of morphine in unanesthetized and anesthetized cats. Br J Pharmacol 26:196-204

14. Holaday JW 1983 Cardiovascular effects of endogenous opiate systems. Annu Rev Pharmacol Toxicol 23:541-594

15. Vatner SF, Franklin D, Braunwald E 1971 Effects of anesthesia and sleep on circulatory response to carotid sinus nerve stimulation. Am J Physiol 220:1249-1255

16. Johnston BM, Gluckman PD 1989 Lateral pontine lesions affect central chemosensitivity in unanesthetized fetal lambs. J Appl Physiol 67:1113-1118 